\title{
Characterizing Families of Cuts That Can Be Represented by Axis-Parallel Rectangles ${ }^{\star}$
}

\author{
Ulrik Brandes ${ }^{1}$, Sabine Cornelsen ${ }^{2}$, and Dorothea Wagner ${ }^{3}$ \\ 1 Fakultät für Mathematik \& Informatik, Universität Passau. \\ brandes@algo.fmi.uni-passau.de \\ 2 Dipartimento di Ingegneria Elettrica, Università dell'Aquila. \\ cornelse@inf .uni-konstanz.de \\ 3 Fakultät für Informatik, Universität Karlsruhe. \\ dwagner@ira.uka.de
}

\begin{abstract}
A drawing of a family of cuts of a graph is an augmented drawing of the graph such that every cut is represented by a simple closed curve and vice versa.

We show that the families of cuts that admit a drawing in which every cut is represented by an axis-parallel rectangle are exactly those that have a cactus model that can be rooted such that edges of the graph that cross a cycle of the cactus point to the root. This includes the family of all minimum cuts of a graph. The proof also yields an efficient algorithm to construct a drawing with axis-parallel rectangles if it exists.
\end{abstract}

\section{Introduction}

A cut of a graph is a partition of its vertex set into two non-empty subsets. In a drawing of a graph, it is therefore natural to represent a cut by a closed curve partitioning the plane into two regions containing one subset each.

When a set of cuts is drawn in this way, the curves can intersect so that their union might contain closed curves that represent other cuts not contained in that set. We are interested in families of cuts that can be drawn without creating confusing non-members. In particular, we consider the problem of drawing families of cuts such that every member is represented by an axis-parallel rectangle and vice versa.

Our main result is a characterization of all families of cuts that can be represented by axis-parallel rectangles, namely those that can be modeled by a cactus containing a certain, rootable, node. These include the important family of all minimum cuts of a graph [4]. We give an algorithm to test this property. Provided a set of cuts of a graph with $n$ vertices and $m$ edges is represented by a cactus model, the test works in $\mathcal{O}(\mathrm{nm})$ time. We also show how to construct a drawing with axis-parallel rectangles if one exists. The construction works in two

\footnotetext{
* Research partially supported by the DFG under grant BR 2158/1-1 and WA 654/131. It was also partially supported by the Human Potential Program of the EU under contract no HPRN-CT-1999-00104 (AMORE Project).
} 
steps. First, we solve the problem for families of minimal cuts of a planar connected graph. Then, we use similar planarization techniques as in [3] to extend the result to general graphs.

The paper is organized as follows. In Sect 2, we define drawings of families of cuts. In Sect. 3, we introduce the cactus model and show that its existence is necessary for the existence of a drawing with axis-parallel rectangles. Using hierarchically clustered graphs (briefly introduced in Sect. 4), we give an additional necessary condition for families of cuts that admit a drawing with axis-parallel rectangles in Section 5 and show how to test it. To show that the conditions are also sufficient, we construct a drawing in Section 6 .

\section{Drawing Families of Cuts}

Throughout this paper, let $G=(V, E)$ denote a simple, connected undirected graph with $n$ vertices and $m$ edges. A drawing $\mathcal{D}$ of $G$ maps vertices on distinct points in the plane and edges on simple curves connecting the drawings of their incident vertices. The interior of an edge must not contain the drawing of a vertex. A drawing is planar, if edges do not intersect but in common end points. Let $S, T \subseteq V$. With $G(S)$ we denote the subgraph of $G$ that is induced by $S$ and with $E(S, T)$ we denote the set of edges that are incident to a vertex in $S$ and a vertex in $T$. A cut of $G$ is a partition $C=\{S, \bar{S}\}$ of the vertex set $V$ into two non-empty subsets $S$ and $\bar{S}:=V \backslash S$. We say that the cut $\{S, \bar{S}\}$ is induced by $S$. The edges in $E(C)=E(S, \bar{S})$ are the cut-edges of $C$. A minimum cut is a cut with the minimum number of cut-edges among all cuts of $G$. A minimal cut is a cut $\{S, \bar{S}\}$ that is inclusion-minimal, i.e. there is no cut $\{T, \bar{T}\}$ with $E(T, \bar{T}) \subsetneq E(S, \bar{S})$. Note that both, $G(S)$ and $G(\bar{S})$, are connected if $\{S, \bar{S}\}$ is a minimal cut of the connected graph $G$.

A drawing of a cut $C=\{S, \bar{S}\}$ of $G$ in a drawing $\mathcal{D}(G)$ is a simple closed curve $\gamma$, such that

- $\gamma$ separates $S$ and $\bar{S}$, i.e. the drawings of edges and vertices in $G(S)$ and $G(\bar{S})$, respectively, are in different connected regions of $\mathbb{R}^{2} \backslash \gamma$, and

- $|\mathcal{D}(e) \cap \gamma|=1$ for $e \in E(C)$, i.e. the drawing of a cut-edge crosses the drawing of the cut exactly once.

Let $\mathcal{C}$ be a set of cuts of a graph $G$. A mapping $\mathcal{D}$ is a (planar) drawing of a graph $G$ and a family of cuts $\mathcal{C}$ of $G$, if

1. $\mathcal{D}(G)$ is a (planar) drawing of $G$ and

2. $\mathcal{D}(C)$ is a drawing of $C$ in $\mathcal{D}(G)$ for every cut $C \in \mathcal{C}$, and

3. every simple closed curve $\gamma \subseteq \bigcup_{C \in \mathcal{C}} \mathcal{D}(C)$ is a drawing of some cut in $\mathcal{C}$.

Note that the third condition eliminates any potential ambiguity regarding which cuts are in the family.

A drawing $\mathcal{D}$ of a graph and a family $\mathcal{C}$ of cuts is a drawing with axisparallel rectangles, if every cut is drawn as an axis-parallel rectangle - more precisely, if every simple closed curve in $\bigcup_{C \in \mathcal{C}} \mathcal{D}(C)$ is an axis-parallel rectangle. See Fig. 1(a) for a planar drawing with axis-parallel rectangles of the set of all minimum and minimum +1 cuts of a graph. 


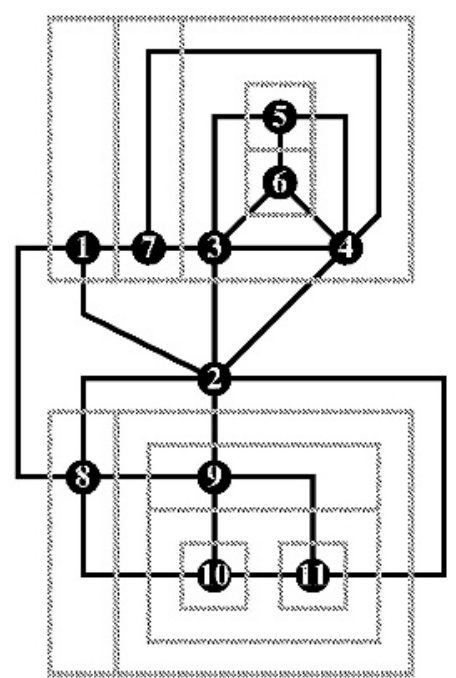

(a) cuts as axis-parallel rectangles

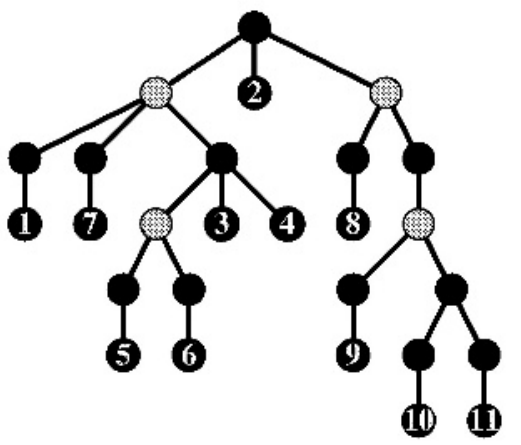

(c) inclusion tree

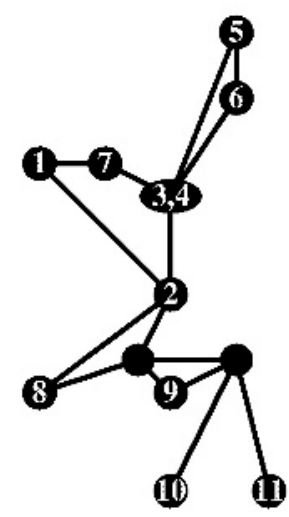

(b) cactus model

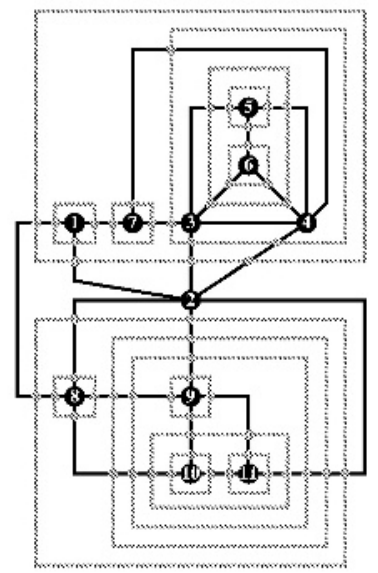

(d) auxiliary graph

Fig. 1. A drawing of the family of all minimum and minimum +1 cuts of a graph with axis-parallel rectangles. In the cactus model of the cuts, $\varphi$ is indicated by node labels, and cycle-replacement nodes in the inclusion tree constructed from the cactus are shown in grey. The auxiliary graph is shown in a c-planar drawing of the hierarchical clustering represented by the inclusion tree.

\section{Necessity of a Cactus Model}

A cactus is a connected graph in which every edge belongs to at most one cycle. A cactus model for a set $\mathcal{C}$ of cuts of a graph $G=(V, E)$ is a pair $(\mathcal{G}, \varphi)$ that 
consists of a cactus $\mathcal{G}=(\mathcal{V}, \mathcal{E})$ and a mapping $\varphi: V \rightarrow \mathcal{V}$ such that $\mathcal{C}$ is modeled by the minimal cuts of $\mathcal{G}$, i.e.,

$$
\mathcal{C}=\left\{\left\{\varphi^{-1}(S), \varphi^{-1}(\bar{S})\right\} ;\{S, \bar{S}\} \text { is a minimal cut of } \mathcal{G}\right\} .
$$

To avoid confusion, the vertices of the cactus $\mathcal{G}$ are called nodes. A node $\nu \in \mathcal{V}$ is called empty if $\varphi^{-1}(\nu)=\emptyset$.

An important family of cuts that has a cactus model is the set of all minimum cuts of a connected graph [4]. A cactus model for the minimum and minimum +1 cuts of the graph in Fig. 1(a) is given in Fig. 1(b).

While not every family of cuts has a cactus model, we show that only those that do can have a drawing with axis-parallel rectangles. Two cuts $\{S, \bar{S}\}$ and $\{T, \bar{T}\}$ cross, if and only if the four corners $S \cap T, S \backslash T, T \backslash S$, and $\overline{S \cup T}$ are non-empty. The four cuts induced by the four corners of two crossing cuts, respectively, are called corner cuts. The cut induced by $(S \backslash T) \cup(T \backslash S)$ is called the diagonal cut.

Theorem 1 ([5]). A set $\mathcal{C}$ of cuts of the graph $G$ can be modeled by a cactus if and only if, for any two crossing cuts $\{S, \bar{S}\}$ and $\{T, \bar{T}\}$ in $\mathcal{C}$,

- the four corner cuts are in $\mathcal{C}$, and

- the diagonal cut is not in $\mathcal{C}$.

If a cactus model exists, there is always one with $O(n)$ nodes.

The properties of crossing cuts in this characterization are implied by overlapping axis-parallel rectangles.

Lemma 1. If a set of cuts has a drawing with axis-parallel rectangles, it has a cactus model.

Proof. Let $\mathcal{D}$ be a drawing of a set $\mathcal{C}$ of cuts with axis-parallel rectangles and suppose that $\mathcal{C}$ contains crossing cuts $\{S, \bar{S}\}$ and $\{T, \bar{T}\}$. There are essentially the seven cases indicated in Fig. 2 for the drawings of two crossing cuts by axisparallel rectangles. Since the cases in Fig. 2(a) contain simple closed curves that are not axis-parallel rectangles, only the case in Fig. 2(b) needs to be considered.

Let $D_{S}, D_{T} \subseteq \mathbb{R}^{2}$ be the rectangular regions bounded by $\mathcal{D}(\{S, \bar{S}\})$ and $\mathcal{D}(\{T, \bar{T}\})$, respectively. Then regions $D_{S} \cap D_{T}, D_{S} \backslash D_{T}, D_{T} \backslash D_{S}$, and $D_{S} \cup D_{T}$ are bounded by axis-parallel rectangles in $\mathcal{D}(\{S, \bar{S}\}) \cup \mathcal{D}(\{T, \bar{T}\})$. These are drawings of the four corner cuts of $\{S, \bar{S}\}$ and $\{T, \bar{T}\}$. Hence, they are in $\mathcal{C}$.

On the other hand, suppose the diagonal cut $C$ induced by $(S \backslash T) \cup(T \backslash S)$ is in $\mathcal{C}$ and let $D_{C}$ be the rectangular region bounded by its drawing $\mathcal{D}(C)$. Without loss of generality, suppose that $\mathcal{D}(S) \subset D_{S}$ and $\mathcal{D}(T) \subset D_{T}$. Either $D_{C}$ contains $\mathcal{D}(G(S \backslash T))$ and $\mathcal{D}(G(T \backslash S))$, but not $\mathcal{D}(G(S \cap T))$, or it contains $\mathcal{D}(G(\overline{(S \backslash T) \cup(T \backslash S)}))$, but not $\mathcal{D}(G(S \backslash T \cup T \backslash S))$. In the first case, the drawing contains a simple closed curve bounding the region $D_{C} \cap D_{S} \cap D_{T}$ (thus inducing an empty cut), and in the second case the union of the three rectangles contains a simple closed curve that is not an axis-parallel rectangle. 

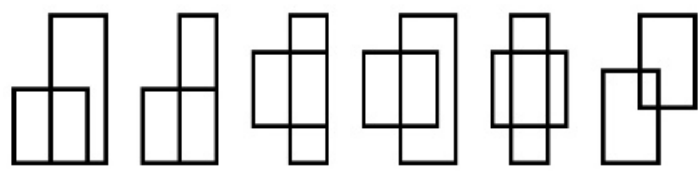

(a) union contains non-axis-parallel rectangles

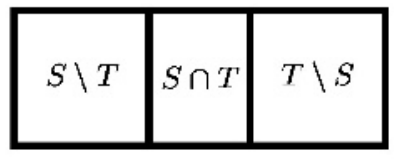

(b) axis-parallel only

Fig. 2. Drawings of two crossing cuts $\{S, \bar{S}\}$ and $\{T, \bar{T}\}$ with axis-parallel rectangles.

\section{Cactus-Induced Hierarchical Clusterings}

Since a cactus model is necessary for a drawing to exist, we can make use of a transformation originally developed for drawing the particular family of all minimum cuts of a planar connected graph [1]. Given a cactus model $(\mathcal{G}, \varphi)$ of a family $\mathcal{C}$ of $G$, we use the tree $T=\mathcal{T}(\mathcal{G}, \varphi)$ that is constructed as follows.

1. Choose a node $r$ of $\mathcal{G}$ as root.

2. Replace each cycle $c$ of $\mathcal{G}$ by a star, i.e. delete every edge of $c$, add a new empty node $\nu_{c}$ - called cycle-replacement node of $c$ - to $\mathcal{G}$, and for every node $\nu$ of $c$, add an edge $\left\{\nu_{c}, \nu\right\}$ to $\mathcal{G}$.

3. For each $v \in V$, add $v$ to the vertex set of $\mathcal{G}$ and add an edge $\{v, \varphi(v)\}$ to $\mathcal{G}$.

The construction is illustrated in Fig. 1(c) and yields a triple $(G, T, r)$ of

- a graph $G=(V, E)$,

- a tree $T$, and

- an inner vertex $r$ of $T$

- where the set of leaves of $T$ is exactly $V$.

This is the hierarchically clustered graph model introduced by Feng et al. [6]. $G$ is called the underlying graph and $T$ the inclusion tree of $(G, T, r)$. Inner vertices of $T$ are called nodes. We denote the tree $T$ rooted at $r$ by $(T, r)$. Each node $\nu$ of $T$ represents the cluster $V_{r}(\nu)$ of leaves in the subtree of $(T, r)$ rooted at $\nu$. Note that $\left\{\left\{V_{r}(\nu), \overline{V_{r}(\nu)}\right\} ; \nu \neq r\right.$ node of $\left.\mathcal{T}(\mathcal{G}, \varphi)\right\}$ equals the subset $\mathcal{C}_{\|}$of non-crossing cuts of $\mathcal{C}$, i.e. the set of cuts in $\mathcal{C}$ that do not cross any other cut in $\mathcal{C}$. A c-planar drawing of a hierarchically clustered graph $(G, T, r)$ consists of

- a planar drawing of the underlying graph $G$ and

- an inclusion representation ${ }^{1}$ of the rooted tree $(T, r)$ such that

- each edge crosses the boundary of the drawing of a node of $T$ at most once.

\footnotetext{
${ }^{1}$ In an inclusion representation of a rooted tree $(T, r)$, each node of $T$ is represented by a simple closed region bounded by a simple closed curve. The drawing of a node or leaf $\nu$ of $T$ is contained in the interior of the region representing a node $\mu$ of $T$ if and only if $\mu$ is contained in the path from $\nu$ to $r$ in $T$. The drawings of two nodes $\mu$ and $\nu$ are disjoint if neither $\mu$ is contained in the path from $\nu$ to $r$ nor $\nu$ is contained in the path from $\mu$ to $r$ in $T$.
} 
Note that the vertices of $G$ are the leaves of $T$ and thus have the same drawing. Also note, that a c-planar drawing of $(G, \mathcal{T}(\mathcal{G}, \varphi), r)$ contains a planar drawing of the set of all non-crossing cuts $\mathcal{C}_{\|}$. A hierarchically clustered graph $(G, T, r)$ is c-planar if it has a c-planar drawing. It is completely connected, if each cluster and the complement of each cluster induces a connected subgraph of $G$. A hierarchically clustered graph with planar underlying graph does not have to be c-planar. However, a completely connected hierarchically clustered graph is c-planar if only the underlying graph is planar [2].

According to the construction in $[1,9]$, we associate an auxiliary graph $G_{\mathcal{D}}$ with a c-planar drawing $\mathcal{D}$ of a hierarchically clustered graph. Let $V^{\prime}$ be the set of points, in which drawings of edges and boundaries of drawings of clusters intersect. Then the vertex set of $G_{\mathcal{D}}$ is $V \cup V^{\prime}$. The edge set of $G_{\mathcal{D}}$ contains two types of edges. For an edge $e=\{v, w\}$ of $G$, let $v_{1}, \ldots, v_{k}$ be the points in $\mathcal{D}(e) \cap$ $V^{\prime}$ in the order they occur in the drawing of $e$ from $v$ to $w$. Then $G_{\mathcal{D}}$ contains the edges $\left\{v, v_{1}\right\},\left\{v_{1}, v_{2}\right\}, \ldots,\left\{v_{k}, w\right\}$. Let $\nu \neq r$ be a node of $T$. Let $v_{1}, \ldots, v_{k}$ be the points in $\partial \mathcal{D}(\nu) \cap V^{\prime}$ in the order they occur in the boundary $\partial \mathcal{D}(\nu)$ of the drawing of $\nu$. Then $G_{\mathcal{D}}$ contains the edges $\left\{v_{1}, v_{2}\right\}, \ldots,\left\{v_{k-1}, v_{k}\right\},\left\{v_{k}, v_{1}\right\}$. The cycle $v_{1}, \ldots, v_{k}$ of $G_{\mathcal{D}}$ is called the boundary cycle of $\nu$. (To avoid loops and parallel edges, additional vertices of degree two may be inserted into boundary cycles). See Fig. 1(d).

\section{Towards a Characterization}

Another necessary condition for families of cuts that have a drawing with axisparallel rectangles depends also on the edges in the graph. Let $(\mathcal{G}, \varphi)$ be the cactus model of a set $\mathcal{C}$ of cuts of a graph $G$. For a cycle $c: \nu_{1}, \ldots, \nu_{k}$ in $\mathcal{G}$ let $V_{i}:=V_{\nu_{c}}\left(\nu_{i}\right), i=1, \ldots, k$. An edge $\{v, w\}$ of $G$ crosses cycle $c$ if there are $1 \leq i, j \leq k$ such that

$$
v \in V_{i}, w \in V_{j} \text {, and } i-j \not \equiv \pm 1 \bmod k .
$$

If $(\mathcal{G}, \varphi)$ is the cactus of all minimum cuts of $G$, then no edge of $G$ crosses a cycle of $\mathcal{G}$. In general, it depends on the edges that cross a cycle of the cactus model, whether a set of cuts has a drawing with axis-parallel rectangles. More precisely, if $\mathcal{C}$ has a drawing $\mathcal{D}$ with axis-parallel rectangles and $e$ crosses the cycle $c$ then there exists an $i \in\{1, \ldots, k\}$ such that $e$ is incident to a vertex in $V_{i}$ and the drawing of $\overline{V_{i}}$ is contained in the simple region bounded by $\mathcal{D}\left(V_{i}, \overline{V_{i}}\right)$. This statement is further formalized in the next lemma.

Lemma 2. A family of cuts that has a cactus model $(\mathcal{G}, \varphi)$ has a drawing with axis-parallel rectangles only if the root $r$ of $\mathcal{T}(\mathcal{G}, \varphi)$ can be chosen such that

(R) each edge of $G$ that crosses a cycle $c$ of $\mathcal{G}$ is incident to a vertex in $V \backslash V_{r}\left(\nu_{c}\right)$.

Proof. Suppose that the set of cuts modeled by $(\mathcal{G}, \varphi)$ has a drawing $\mathcal{D}$ with axis-parallel rectangles. Let $r$ be a node of $T=\mathcal{T}(\mathcal{G}, \varphi)$ such that for every node $\nu \neq r$ of $T$ the set $V_{r}(\nu)$ is contained in the simple region bounded by 
$\mathcal{D}\left(\left\{V_{r}(\nu), \overline{V_{r}(\nu)}\right\}\right)$. Using the fact that $\overline{V_{r}(\mu)}=V_{\mu}(r)$ for two adjacent nodes $r$ and $\mu$ of $T$, it can be shown that such a node $r$ exists.

Let $c$ be a cycle of $\mathcal{G}$ and let $e$ be an edge of $G$ that crosses $c$. Let $\nu_{1}, \ldots, \nu_{k}$ be the descendents of $\nu_{c}$ such that $\nu_{1}, \ldots, \nu_{k}$ is a path in $c$. Suppose that $e$ is not incident to a vertex in $V \backslash V_{r}\left(\nu_{c}\right)$. Then $e \in E\left(V_{r}\left(\nu_{i}\right), V_{r}\left(\nu_{j}\right)\right)$ for some $1<i+1<j \leq k$. Let $S=\bigcup_{\ell=1}^{i+1} V_{r}\left(\nu_{\ell}\right)$ and $T=\bigcup_{\ell=i+1}^{k} V_{r}\left(\nu_{\ell}\right)$. Then, on one hand, $e \in E(S \backslash T, T \backslash S)$. But, on the other hand, $\{S, \bar{S}\}$ and $\{T, \bar{T}\}$ are two crossing cuts that are modeled by $(\mathcal{G}, \varphi)$ such that $S$ and $T$ are contained in the simple closed region $D_{S}$ and $D_{T}$ bounded by $\mathcal{D}(\{S, \bar{S}\})$ and $\mathcal{D}(\{T, \bar{T}\})$, respectively. Hence, we have again the situation as indicated in Fig. 2(b). By the definition of drawings of cuts, the drawing of the edges and vertices of $G(S \cup T)$ has to be contained inside $D_{S} \cup D_{T}$ and the drawing of the edges and vertices of $\mathcal{D}(G(\overline{S \cap T}))$ may not intersect $D_{S} \cap D_{T}$. This implies that $E(S \backslash T, T \backslash S)=\emptyset$.

In the following, a node of the inclusion tree $T=\mathcal{T}(\mathcal{G}, \varphi)$ is called rootable, if it fulfills Condition ( $\mathrm{R}$ ) of the previous lemma. We give an algorithm for finding all rootable nodes of the inclusion tree $T=\mathcal{T}(\mathcal{G}, \varphi)$. We assume that the size of $\mathcal{G}$ is in $\mathcal{O}(n)$.

We use a node and edge array CROSSED on $T$ to store the information about edges of $G$ that cross a cycle of the cactus. Let $c$ be a cycle of $\mathcal{G} \cdot \operatorname{CrOsSED}\left(\nu_{c}\right)$ is true if and only if there is an edge of $G$ that crosses $c \cdot \operatorname{CROSSED}\left(\left\{\nu_{c}, \nu\right\}\right)$ is true for an edge $\left\{\nu_{c}, \nu\right\}$ of $T$ if and only if $\operatorname{CROSSED}\left(\nu_{c}\right)$ is true and each edge that crosses $c$ is incident to a vertex in $V_{\nu_{c}}(\nu)$. For all other edges and nodes of $T$ CROssed is false. Clearly, it can be tested in linear time, which cycles are crossed by an edge. Hence, CROSSED can be computed in $\mathcal{O}(m n)$ time. The next lemma is a reformulation of Condition $(\mathrm{R})$.

Lemma 3. A node $r$ of $T$ is rootable if and only if for each node $\nu$ of $T$ with $\operatorname{CROSSED}(\nu)=$ TRUE there exists an adjacent node $\mu$ of $\nu$ with $\operatorname{CROSSED}\{\nu, \mu\}=$ TRUE such that $r$ is contained in the subtree of $(T, \nu)$ rooted at $\mu$.

Hence, we can obtain the possible roots for constructing a drawing with axisparallel rectangles of $\mathcal{C}$ - possibly including some cycle-replacement nodes - by deleting for all marked nodes $\nu$ the subtrees of $(T, \nu)$ rooted at those adjacent nodes $\mu$ of $\nu$ for which $\{\nu, \mu\}$ is not marked. Proceeding first from the leaves to an arbitrary root and then from this root to the leaves of $T$, this can be done in time $\mathcal{O}(n)$.

By constructing a drawing, we show in the next section that the conditions in Lemma 2 are also sufficient.

\section{The Drawing}

Let $(\mathcal{G}, \varphi)$ continue to be a cactus model of a set $\mathcal{C}$ of cuts of the graph $G$ and let $T=\mathcal{T}(\mathcal{G}, \varphi)$ be the inclusion tree constructed in Sect. 4 . We assume that the root $r$ of $T$ is a rootable node. We show how to construct a drawing with 
axis-parallel rectangles for $\mathcal{C}$. In a first step, we consider the case that $\mathcal{C}$ is a set of minimal cuts of a connected planar graph. Using planarization, we generalize the result to general sets of cuts of not necessarily planar graphs.

\subsection{Planar Graphs}

In this section, we assume that $\mathcal{C}$ is a set of minimal cuts of a planar connected graph $G$. We show how the ideas for drawing the set of all minimum cuts of a planar graph [1] yield a drawing with axis-parallel rectangles of $\mathcal{C}$. We briefly sketch the general construction and explain in more details the parts that differ from the case of drawings for the set of minimum cuts. The construction starts with a c-planar drawing $\mathcal{D}$ of the hierarchically clustered graph $(G, T, r)$. Such a c-planar drawing always exists: Since $\mathcal{C}$ is a set of minimal cuts of a connected graph it follows that $(G, T, r)$ is completely connected and hence c-planar.

Let $c: \nu_{1}, \ldots, \nu_{\ell}$ be a cycle of $\mathcal{G}$. Suppose that $\nu_{c}$ is a descendent of $\nu_{\ell}$ in the tree $T$ rooted at $r$. Let $V_{i}=V_{\nu_{c}}\left(\nu_{i}\right), i=1, \ldots, \ell$ and let $V_{0}=V_{\ell}$. Let $i \in\{1, \ldots, \ell-1\}$ and let $e_{1}, \ldots, e_{k}$ be the sequence of edges incident to a vertex in $V_{i}$ in the cyclic order around $V_{i}$. Then $E\left(V_{i}, V_{i-1}\right)$ and $E\left(V_{i}, V_{i+1}\right)$ are non-empty subsequences of $e_{1}, \ldots, e_{k}$, i.e. suppose $e_{1} \in E\left(V_{i}, V_{i-1}\right)$ and $e_{k} \notin E\left(V_{i}, V_{i-1}\right)$, then there are indices $1<k_{1} \leq k_{2}<k_{3} \leq k$ such that

$$
\underbrace{e_{1}, \ldots, e_{k_{1}}}_{E\left(V_{i}, V_{i-1}\right)}, \underbrace{e_{k_{1}+1}, \ldots, e_{k_{2}}}_{E\left(V_{i}, V_{\ell}\right)}, \underbrace{e_{k_{2}+1}, \ldots, e_{k_{3}}}_{E\left(V_{i}, V_{i+1}\right)}, \underbrace{e_{k_{3}+1}, \ldots, e_{k}}_{E\left(V_{i}, V_{\ell}\right)} .
$$

Further, $E\left(V_{\ell}, V_{1}\right)$ and $E\left(V_{\ell}, V_{\ell-1}\right)$ are non-empty subsequence of the edges around $V \backslash V_{\ell}$. This observation can be proved in the following two steps.

1. Since $G$ is connected and $\mathcal{C}$ is a set of minimal cuts, it follows that the sets $E\left(V_{i}, V_{i-1}\right), E\left(V_{i}, V_{i+1}\right), i=1, \ldots, \ell-1$ are non-empty.

2. It follows from Step 1 and the c-planarity that $E\left(V_{i}, V_{i-1}\right), E\left(V_{i}, V_{i+1}\right), i=$ $1, \ldots, \ell-1$ are subsequences of the cyclic sequence of edges around $V_{i}$ and that $E\left(V_{\ell}, V_{1}\right), E\left(V_{\ell}, V_{\ell-1}\right)$ are subsequences of the cyclic sequence of edges around $V \backslash V_{\ell}$.

Besides, let $e \in E\left(V_{i}, V_{j}\right)$ for some $i, j \in\{1, \ldots, \ell\}, i \neq j$. Then c-planarity implies that the two vertices in the auxiliary graph $G_{\mathcal{D}}$ that represent the intersection of $e$ with the boundary of $\nu_{i}$ and $\nu_{j}$ (or the boundary of $\nu_{c}$, if $i=\ell$ or $j=\ell$ ), respectively, are adjacent. Hence the situation in the auxiliary graph $G_{\mathcal{D}}$ is as indicated in Fig. 3a. For $i \in\{1, \ldots, \ell-1\}$, let $p_{i}^{ \pm}$be the path in $G_{\mathcal{D}}$ that is induced by the intersection of the edges in $E\left(V_{i}, V_{i \pm 1}\right)$ with the boundary of $\nu_{i}$. Similarly, let $p_{0}^{+}\left(p_{\ell}^{-}\right)$be the path in $G_{\mathcal{D}}$ that is induced by the intersection of the edges in $E\left(V_{\ell}, V_{1}\right)\left(E\left(V_{\ell}, V_{\ell-1}\right)\right)$ with the boundary of $\nu_{c}$.

A planar drawing of $\mathcal{C}$ with axis-parallel rectangles can now be obtained as follows. First, for each $i$, paths $p_{i}^{+}$and $p_{i+1}^{-}$are united to one path. The parts of the boundary-cycle of $\nu_{c}$ that are not in $p_{0}^{+}$or $p_{\ell}^{-}$are removed. The result can be seen in Fig. 3b. Finally, at each end of each thus united path $p_{i}^{+} / p_{i+1}^{-}$, 
$i=1, \ldots, \ell-2$, an additional vertex is inserted as indicated in Fig. 3c. In the following, we refer to these vertices as cycle-path end-vertices. Let $G^{\prime}$ be the graph that results from $G_{\mathcal{D}}$ by doing this for every cycle of $\mathcal{G}$. Applying a min-cost flow approach for quasi-orthogonal drawings $[10,8,7,11]$ with some restriction on the flow to $G^{\prime}$ yields a drawing of $\mathcal{C}$ with axis-parallel rectangles. There are two restrictions on the flow necessary

1. the flow over a boundary edge from outside a boundary cycle into the inside of a boundary cycle is zero

2. the flow from a cycle-path end-vertex into the inside of a boundary cycle is minimum, i.e. there is a rectangle.

It can be shown that there is always a feasible flow for the thus restricted flow network.

a)

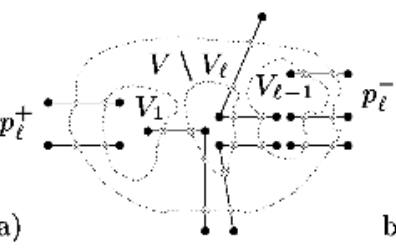

b)

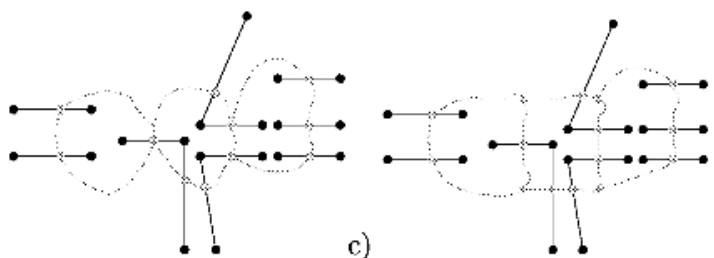

c)

Fig. 3. Constructing a drawing for a set of cuts from a c-planar drawing.

\subsection{General Graphs}

In this section, we extend the results on planar drawings for families of minimal cuts to not necessarily planar drawings. Similar to the method described in [3] for hierarchically clustered graphs, the idea for the construction uses planarization techniques. Recall that $(\mathcal{G}, \varphi)$ is a cactus model for a family of cuts of $G$. We assume again that the root $r$ of $T=\mathcal{T}(\mathcal{G}, \varphi)$ is rootable.

1. Let $G_{P}=\left(V, E_{P}\right)$ be a planar connected graph on the same set of vertices as $G$, such that $\mathcal{G}$ models a set of minimal cuts and each edge of $G_{P}$ that crosses a cycle $c$ of $\mathcal{G}$ is incident to a vertex in $V \backslash V_{r}\left(\nu_{c}\right)$.

2. Construct the auxiliary graph $G_{P}^{\prime}$ for $\left(G_{P}, \mathcal{G}, \varphi\right)$ as it is described in Sect. 6.1.

3. Construct a planar graph $G^{\prime}$, by inserting the remaining edges of $G$ into $G_{P}^{\prime}$ such that each edge crosses the boundary cycle of a cut at most once.

4. Apply an algorithm for quasi-orthogonal drawings with the restrictions indicated in Sect. 6.1 to $G^{\prime}$.

5. Delete the edges that do not correspond to original edges in $G$. 
Remarks to Step 1. It is not always possible to choose the graph $G_{P}$ with the properties required in Step 1 as a subgraph of $G$. The following lemma shows, however, that at least some graph with the properties required in Step 1 exists.

Lemma 4. Let $(\mathcal{G}, \varphi)$ be a cactus model for a set of cuts of a graph with vertex set $V$. Then there exists a planar connected graph $H$ with vertex set $V$ such that

1. $\mathcal{G}$ models a set of minimal cuts of $H$ and

2. no edge of $H$ crosses a cycle of $\mathcal{G}$.

Proof. Let $\mu$ be a node of $T$ that is adjacent to a leaf $v$. In a first step, we start with an empty graph. Proceeding from bottom to top of the tree $T$ rooted at $\mu$, we can construct a graph $H_{0}=\left(V, E_{0}\right)$ such that

1. for any node $\nu \neq \mu$ the subgraph of $H_{0}$ induced by $V_{\mu}(\nu)$ is a path,

2. no edge of $H_{0}$ crosses a cycle of $\mathcal{G}$, and

3. $E_{0}\left(V_{\mu}(\nu), \overline{V_{\mu}(\nu)}\right)=\emptyset$ if $\nu$ is adjacent to $\mu$.

In a second step, for each node $\nu$ of $T$ that is adjacent to $\mu$ let $v_{1}$ and $v_{2}$ be the two vertices of degree one or let $v_{1}=v_{2}$ be the vertex of degree zero in the subgraph of $H_{0}$ induced by $V_{\mu}(\nu)$, respectively. Add edges $\left\{v, v_{1}\right\}$ and $\left\{v, v_{2}\right\}$ to $H_{0}$. Finally, for all leaves $w \neq v$ of $T$ that are adjacent to $r$, add an edge $\{v, w\}$ to $H_{0}$.

It is now easy to see that no edge of the thus constructed graph $H$ crosses a cycle of $\mathcal{G}$ and that $\mathcal{G}$ models a set of minimal cuts. $H$ is a cactus and hence it is planar and connected.

Let $H=\left(V, E_{H}\right)$ be the graph from the previous lemma and let $G_{P}$ be a maximal planar subgraph of $\left(V, E \cup E_{H}\right)$ such that $H$ is a subgraph of $G_{P}$. Then $G_{P}$ is planar and connected, $\mathcal{G}$ models a set of minimal cuts of $G_{P}$ and $r$ is rootable. Hence, $G_{P}$ fulfills the properties required in Step 1.

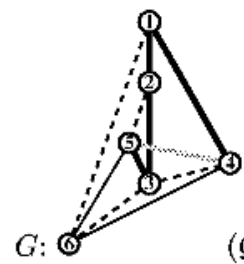

$(\mathcal{G}, \varphi):$
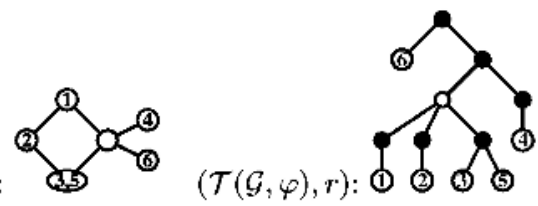

Fig. 4. A non-planar graph $G$ and a cactus model. Deleting the grey edge yields a maximal planar subgraph. Solid thick black edges induce a spanning path in every cluster $S \neq V$ of $(G, \mathcal{T}(\mathcal{G}, \varphi), r)$. All solid black edges indicate the graph $H$ constructed in the proof of Lemma 4 . 
Remarks to Step 3. We use an extention of planarization-techniques that is similar to the method introduced by Di Battista et al. [3] for drawing non-planar hierarchically clustered graphs. Edges in $G$ that are not represented in $G_{P}$ are routed iteratively through the dual graph of $G_{P}^{\prime}$, replacing every crossing of an edge and a dual in the rout by a vertex of degree four. Suppose, we want to insert an edge $\{v, w\}$. Let $F$ be the set of faces of $G_{P}^{\prime}$. Let $E_{\{v, w\}}^{*}$ be the following set of arcs. For every edge $e$ of $G_{P}^{\prime}$, let $f_{1}, f_{2} \in F$ be the faces that are incident to $e$. Then $E_{\{v, w\}}^{*}$ contains the dual arcs $\left(f_{1}, f_{2}\right)_{e}$ and $\left(f_{2}, f_{1}\right)_{e}$. Further, $E_{\{v, w\}}^{*}$ contains the $\operatorname{arcs}(f, x),(x, f), x \in\{v, w\}, f \in F, f$ incident to $x$. There are no other arcs contained in $E_{\{v, w\}}^{*}$. In general, any simple path in the extended dual $G_{\{v, w\}}^{*}=\left(\{v, w\} \cup F, E_{\{v, w\}}^{*}\right)$ from $v$ to $w$ can be used as a rout for the edge $\{v, w\}$. To achieve that the drawing of $\{v, w\}$ does not cross a boundary cycle twice, we use a restricted version of the extended dual. Let $v=\nu_{1}, \nu_{2} \ldots, \nu_{\ell}=w$ be the path in $T$ between $v$ and $w$. Let $k \in\{2, \ldots, \ell-1\}$ be such that $\nu_{k-1}$ and $\nu_{k+1}$ are descendants of $\nu_{k}$. For each boundary edge $e$ of $G_{P}^{\prime}$,

- if $e$ is contained in the drawing $\gamma$ of $\left\{V_{r}\left(\nu_{i}\right), \overline{V_{r}\left(\nu_{i}\right)}\right\}, i=2, \ldots, k-1$, delete the dual arc of $e$ that is directed from the outside of $\gamma$ to the inside of $\gamma$ from $E_{\{v, w\}}^{*}$.

- if $e$ is contained in the drawing $\gamma$ of $\left\{V_{r}\left(\nu_{i}\right), \overline{V_{r}\left(\nu_{i}\right)}\right\}, i=k+1, \ldots, \ell-1$, delete the dual arc of $e$ that is directed from the inside of $\gamma$ to the outside of $\gamma$ from $E_{\{v, w\}}^{*}$.

- if $e$ is contained in the drawing of a cut $\left\{V_{r}(\nu), \overline{V_{r}(\nu)}\right\}$ for a node $\nu \neq \nu_{i}, i=$ $2, \ldots, k-1, k+1, \ldots, \ell-1$ of $T$, delete both dual arcs of $e$ from $E_{\{v, w\}}^{*}$.

a)

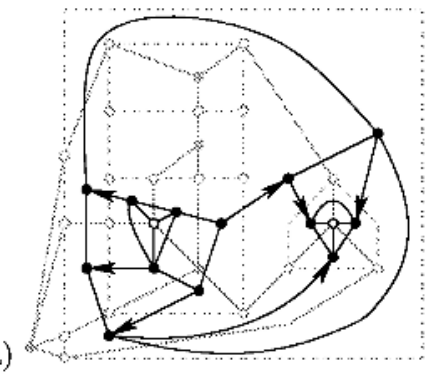

b)

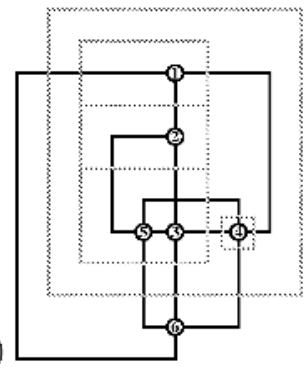

Fig. 5. a) Black edges show a connected component of the restricted extended dual for adding the edge $\{5,4\}$ from 5 to 4 of the graph in Fig. 4. Bidirected edges are indicated by simple curves without arrows. b) A drawing with axis-parallel rectangles for the family of cuts modeled by $(\mathcal{G}, \varphi)$ in Fig. 4 .

The thus restricted dual (see Fig. 5 for an illustration) guarantees that any path from $v$ to $w$ crosses any boundary cycle at most once. On the other hand, there 
is a path from $v$ to $w$ in the restricted dual if and only if either $\nu_{k}$ is not a cyclereplacement node or $\nu_{k}$ is a cycle-replacement node and $\nu_{k-1}, \nu_{k+1}$ are adjacent in $\mathcal{G}$. Hence, by Condition $(\mathrm{R})$, there is a path from $v$ to $w$. Summarizing, we have shown the following characterization

Theorem 2. A family $\mathcal{C}$ of (minimal) cuts of a (planar) graph $G$ has a (planar) drawing with axis-parallel rectangles if and only if

1. $\mathcal{C}$ has a cactus model $(\mathcal{G}, \varphi)$ and

2. the root $r$ of $\mathcal{T}(\mathcal{G}, \varphi)$ can be chosen such that each edge that crosses a cycle $c$ is incident to a vertex in $V \backslash V_{r}\left(\nu_{c}\right)$.

Provided a cactus model is given, it can be tested in time $\mathcal{O}(\mathrm{nm})$ whether a drawing with axis-parallel rectangles exists.

\section{References}

1. U. Brandes, S. Cornelsen, and D. Wagner. How to draw the minimum cuts of a planar graph. In J. Marks, editor, Proceedings of the 8th International Symposium on Graph Drawing (GD 2000), volume 1984 of Lecture Notes in Computer Science, pages 103-114. Springer, 2001.

2. S. Cornelsen and D. Wagner. Completely connected clustered graphs. To appear in Proceedings of the 29th International Workshop on Graph Theoretic Concepts in Computer Science (WG 2003).

3. G. Di Battista, W. Didimo, and A. Marcandalli. Planarization of clustered graphs. In M. Jünger and P. Mutzel, editors, Proceedings of the 9th International Symposium on Graph Drawing (GD 2001), volume 2265 of Lecture Notes in Computer Science, pages 60-74. Springer, 2002.

4. Y. Dinitz, A. V. Karzanov, and M. Lomonosov. On the structure of a family of minimal weighted cuts in a graph. In A. Fridman, editor, Studies in Discrete Optimization, pages 290-306. Nauka, 1976. (in Russian).

5. Y. Dinitz and Z. Nutov. A 2-level cactus model for the system of minimum and minimum +1 edge-cuts in a graph and its incremental maintenance. In Proceedings of the 27th Annual ACM Symposium on the Theory of Computing (STOC '95), pages 509-518. ACM, The Association for Computing Machinery, 1995.

6. Q. Feng, R. F. Cohen, and P. Eades. Planarity for clustered graphs. In P. Spirakis, editor, Proceedings of the 3rd European Symposium on Algorithms (ESA '95), volume 979 of Lecture Notes in Computer Science, pages 213-226. Springer, 1995.

7. U. Fößmeier and M. Kaufmann. Drawing high degree graphs with low bend numbers. In F. J. Brandenburg, editor, Proceedings of the 3rd International Symposium on Graph Drawing (GD '95), volume 1027 of Lecture Notes in Computer Science, pages 254-266. Springer, 1996.

8. G. W. Klau and P. Mutzel. Quasi orthogonal drawing of planar graphs. Technical Report MPI-I-98-1-013, Max-Planck-Institut für Informatik, Saarbrücken, Germany, 1998. Available at http://data.mpi-sb.mpg.de/internet/reports.nsf.

9. D. Lütke-Hüttmann. Knickminimales Zeichnen 4-planarer Clustergraphen. Master's thesis, Universität des Saarlandes, 1999. (Diplomarbeit).

10. R. Tamassia. On embedding a graph in the grid with the minimum number of bends. SIAM Journal on Computing, 16:421-444, 1987.

11. R. Tamassia, G. Di Battista, and C. Batini. Automatic graph drawing and readability of diagrams. IEEE Transactions on Systems, Man and Cybernetics, 18(1):61-79, 1988. 